\title{
ULTRA-DILUTED VISCUM ALBUM IN THE TREATMENT OF CUTANEOUS MELANOMA IN A DOG (CANIS FAIMILIARIS) - CASE REPORT
}

KEYWORD:Cancer, Mistletoe, Complementary Therapy

\section{Ana Catarina Viana Valle*}

\section{Aloísio Cunha de Carvalho}

Doctor Izao Soares Institute, Integrative Medicine. Ribeirao Preto, Brazil. *Corresponding Author

Doctor Izao Soares Institute, Integrative Medicine. Ribeirao Preto, Brazil.

Background: Cutaneous melanoma is one of the tumors with the highest incidence in middle-aged dogs. Effective systemic treatments for advanced malignant melanoma are lacking, and survival is about 1 to 3 months. Viscum album therapy is among the complementary therapies against cancer. Methods: A 7-year-old Dogue de Bordeaux female dog. The main complaint was an ulcerated lesion in the right anterior leg. The animal had already been diagnosed with malignant cutaneous melanoma 11 months before. Extensive ulcerated lesions in the fourth digit of the right anterior leg, mandibular region, and labial commissures were observed for more than nine months, with no resolution of the initial condition. Pulmonary metastasis in the left caudal lobe, two evolving breast tumors, and several blackish nodular lesions spread through the abdomen, lower eyelids, and auricular pavilions were already diagnosed. An injectable, homeopathic treatment was prescribed and consisted of an intravenous application of Viscum album D3 $\left(1 \times 10^{-3}\right)$ and auto-hemotherapy, once a week. At home, the tutor performed applications of Viscum album D3 $\left(1 \times 10^{-3}\right), \mathrm{D} 6\left(1 \times 10^{-6}\right), \mathrm{D} 12$ $\left(1 \times 10^{-12}\right), \mathrm{D} 30\left(1 \times 10^{-30}\right)$, in combinations, SID, subcutaneously, three times a week. Conclusion: The protocol administered to the patient in this study was successful in its purpose. It healed the wounds and improved the patient's quality of life. Other studies must be conducted, under the same conditions, to better elucidate the results obtained in the present study.

\section{INTRODUCTION}

Melanocyte tumors represent $11 \%$ of all skin cancers in small animals. Among these, approximately $7 \%$ are tumors considered to be malignant, while $4 \%$ are benign neoplasms (Lima et al., 2018). Additionally, these tumors are reported with a diagnostic frequency of $70 \%$ and $30 \%$, respectively, among all melanocyte tumors (Gillard et al., 2013).

Melanoma is the most common cancer of the oral cavity of dogs (Wobeser et al., 2007), representing $62 \%$ of the total diagnosed melanomas. The most common primary sites of these tumors in small animals are the oral mucosa, eyes, nail region, skin in general (Inoue et al., 2004). It is considered the second most prevalent in the digits region (Manley et al., 2011). According to Wobeser et al. (2007), approximately $53 \%$ of all lesions affecting dogs' digits are malignant neoplasms. Squamous cell carcinoma and melanoma are the main histological types (Manley et al., 2011).

Patients affected by melanoma are, in general, dogs and cats between 8 and 12 years old. There is no sex predilection in both species. However, some dog breeds are more affected, such as Cocker Spaniel, Scottish Terrier, Boston Terrier, Airedale Terrier, Schnauzer, Doberman, Pincher, Vizlas, Golden Retriever, Irish Setter, Chowchow, and Boxer, in addition to mixed-breed dogs. On the other hand, in the feline species, melanoma is more common in cats with black or gray fur (Grandi and Rondelli, 2016).

The occurrence of metastases is quite common, with regional lymph nodes and lungs as the most frequent sites, which can metastasize to the brain, heart, spleen, and, less frequently, liver. Besides, it is usually a tumor with a high probability of recurrence and tissue invasion (Bolon et al., 1990). The survival time of the affected animals after diagnosis is usually 6-12 months (Gillard et al., 2013; Rodrigues et al.,2017).

The diagnosis occurs through the macro and microscopic characteristics of the neoplasm, physical examination, associated with complementary exams, such as cytopathology, histopathology (Sulaimon et al., 2002), and immunohistochemistry.

|www.worldwidejournals.com
The treatment of choice for melanoma is, when possible, the surgical removal of the neoplasia, considering the safety margins. As an example, dermal melanomas are generally benign and often cured by surgical excision in dogs. However, mucous and mucocutaneous melanomas are aggressive tumors with a high metastasis rate to regional lymph nodes and lung parenchyma (Wobeser et al., 2007). Although surgery or radiation therapy can be effective in local disease control, effective systemic treatments for advanced malignant melanoma are lacking. Consequently, survival is about 1 to 3 months (Boston et al., 2014; Tuohy et al., 2014). Therefore, the prognosis in dogs is no different from that in humans, for whom the prognosis for stage IV melanoma is poor, with an average survival time of $6-9$ months. Survival is only $32-63 \%$ in 1 year and 15-20\% in 5 years (Siegel et al.,2016).

Viscum album therapy is among the complementary therapies against cancer. It has been used for more than 100 years for this purpose, according to Anthroposophical Medicine. This therapy is indicated for treating cancer patients in a curative and/or palliative way with increasing frequency (Kaestner et al., 2019; Menke et al., 2019). It is considered the most frequently prescribed medication among German doctors (Rostock, 2020; Matthes et al., 2020), including among pediatricians (Menke et al., 2019). In Europe, more than $88 \%$ of cancer patients opt for complementary therapies, and $77 \%$ of them use therapy with Viscum album (Matthes et al. , 2020).

The effectiveness of complementary supportive treatments, such as Viscum album, is of particular interest. Viscum album extracts are often used in integrative medicine to improve life quality and reduce adverse side effects (Kienle and Kiene, 2010).Viscum album applications are generally well tolerated and have few and mild side effects (Steele et al., 2015).

Different authors have already reported the efficacy of the Viscum album administration for treating cutaneous melanoma (Struh et al., 2012; Worthmann et al., 2017; Melo et al., 2018; Valle et al., 2020). Worthmann et al. (2017) reported excellent results in a human patient with malignant cutaneous melanoma and metastasis. The patient was treated using 
Viscum album therapy and had a long-term associated with excellent survival. However, this patient's treatment was instituted as soon as a conclusive diagnosis was made, implying a better prognosis with this therapy.

Valle et al. (2020) also reported the cutaneous melanoma treatment in a canine, in which the neoplastic lesion was ulcerated. The Viscum album therapy was started, and the wound healed within four weeks. The patient was followed up for more than eight months without recurrence or metastasis.

Therefore, this work aims to report the treatment of a cutaneous melanoma case in a 7-year-old Dogue de Bordeaux. The female dog was diagnosed with a cutaneous melanoma 11 months before. It was resistant to conventional chemotherapy melanoma and was treated by the Viscum album therapy for ten weeks.

\section{CASE DETAILS}

A 7-year-old Dogue de Bordeaux female dog (Fig. 1 - A) was attended at the NaturalPet Clinic in Brasilia, Brazil. The dog weighed $32 \mathrm{Kg}$ and was fed with commercial feed. She had no history of vomiting, diarrhea, dyspnea or apathy, and lack of appetite. The main complaint was an ulcerated lesion in the right anterior leg (Fig. 1 - B). The animal had already been diagnosed with malignant cutaneous melanoma 11 months before, and no surgical excision of any region was performed. Extensive ulcerated lesions in the fourth digit of the right anterior leg, mandibular region, and labial commissures (Fig. $2-$ A) were observed for more than nine months, with no resolution of the initial condition. The submandibular, inguinal, and popliteal lymph nodes were infarcted. Nevertheless, the tutor reported having performed four cycles of conventional chemotherapy with no success. Pulmonary metastasis in the left caudal lobe, two evolving breast tumors, and several blackish nodular lesions spread through the abdomen (Fig. 2 - C), lower eyelids (Fig. 2 - B), and auricular pavilions were already diagnosed. At the physical examination, the patient was cheerful, alert to stimuli, had normal mucous membranes, CRT 2", lack of sensitivity to abdominal palpation, normal cardiac auscultation according to age and species. The tutor's main complaints were confirmed, such as ulcerated lesion in the fourth digit, bilateral ulcerated lesion in the labial commissures, and left cranial portion of the mandible. Breast tumors in M5E and M3D and melanocytic pattern nodules disseminated through the abdomen (Fig. 2 - C) of approximately $2 x 1.2 \mathrm{~cm}$ and $0.9 \times 1.5 \mathrm{~cm}$ were also confirmed. Blood was collected for complete blood count and biochemical measurements of urea, creatinine, alanine aminotransferase, and alkaline phosphatase. The animal was prescribed the oral Vitamin D3 2000UI, one capsule, SID, 30 days; Vitamin C $500 \mathrm{mg}$, one tablet, SID, 30 days; Phosphorus $30 \mathrm{CH}\left(1 \times 10^{-30}\right)-30 \mathrm{~mL}, 10 \%$ alcohol, four drops, two times a day, for 30 days; and low-carb diet ( $10 \%$ carbohydrate). An injectable, homeopathic treatment was prescribed and consisted of an intravenous application of Viscum album D3 $\left(1 \times 10^{-3}\right)$ and autohemotherapy, once a week, at the clinic. At home, the tutor performed applications of Viscum album D3 $\left(1 \times 10^{-3}\right)$, D6 $\left(1 \times 10^{-6}\right), \mathrm{D} 12\left(1 \times 10^{-12}\right), \mathrm{D} 30\left(1 \times 10^{-30}\right)$, in combinations, SID, subcutaneously, three times a week.

\section{DISCUSSION}

Malignant melanoma is among the skin neoplasms with the highest incidence in dogs. This neoplasm is characterized by presenting pigmented nodules, which can be located on the face, trunk, legs, mucocutaneous transitions, nail region, and scrotum (Ettinger and Feldman, 2004). This description is in line with the case here reported, where it is demonstrated the various nodular lesions with melanocytic pattern disseminated in the patient's body and subsequent diagnosis of the disease.
The animal was treated over ten weeks, subcutaneously and intravenously, starting on October $4^{\text {th }}$ and ending on December $5^{\text {th }}$, presenting total wound healing. The lesions of the mandible and labial commissures were the first to heal (Fig. 3 - E). However, complete healing of the epithelium occurred over four weeks (Fig. 3). The ulcerated lesion of the paw occurred over ten whole weeks (Fig. 4). Blood tests showed the follow-up and minimal changes in the patient's biochemical and hematological parameters (Table 1). Since the first week, right after the first application, the tutor reported that the animal improved its overall condition, appetite, presented regular sleep, had started playing again, and was more stable and active.

This information corroborates Oei et al. (2020), who recorded the life quality improvement in breast cancer patients in various stages when they used the Viscum album therapy. Our findings are also corroborated by Valle et al. (2020), who reported a cutaneous melanoma in a dog treated with Viscum album therapy, using a similar protocol, with recovery and subsequent follow-up for eight months of the patient.

However, after the complete healing of the wound in the right anterior leg, the patient did not return to the clinic for the intravenous application. The tutor preferred to perform only the subcutaneous applications at home, discontinuing the protocol with the intravenous applications. At 14 days after the protocol discontinuation (December $19^{\text {th }}$ ), the patient returned to the clinic, and the tutor complained of dyspnea and lack of appetite during the past 24 hours. An x-ray was requested (Fig. 5), and pleural effusion was verified. The animal died 12 hours after diagnosis.

Malignant cutaneous melanoma is a disease of complex treatment because of patients with inoperable diseases or metastatic alterations since the conventional clinical treatment does not include the full disease resolution, nor does it even guarantee a better quality of life. When neoplasia is at an advanced stage, the patient's comfort and quality of life become essential palliative care. In this context, complementary treatments, such as the Viscum album therapy, are important tools for enhancing the treatment quality of such animals with no hope of improving their overall condition.

The conventional treatment model does not provide quality of life to patients in advanced stages of cancer. Therefore, the feeling of well-being of these individuals is reduced. However, the Viscum album therapy can provide several benefits to these patients, even when death is close, as abovementioned for the patient cited in this report, who benefited from the palliative care from the Viscum album therapy. In this context, despite the poor prognosis of the disease, the subcutaneous and intravenous protocol allowed wound healing and the patient's quality of life improvement.

Therefore, the protocol administered to the patient in this study was successful in its purpose. It healed the wounds and improved the patient's quality of life until the day of its death. Other studies must be conducted, under the same conditions, to better elucidate the results obtained in the present study.

\section{CONFLICT OF INTEREST}

The authors declare that they have no conflict of interest.

Table 1 - Description Of The Patient's Laboratory Parameters At The Beginning And The End Of The Treatment.

\begin{tabular}{|c|c|c|}
\hline Laboratory Tests & October $^{\text {th }}$ & December $19^{\text {th }}$ \\
\hline Blood count (uL) & $7,160,000$ & $5,760,000$ \\
\hline Hemoglobin $(\mathrm{g} / \mathrm{dL})$ & 16 & 12.6 \\
\hline Hematocrit $(\%)$ & 46.8 & 37 \\
\hline MCV (fL) & 64.64 & 64.24 \\
\hline & www.worldwidejournals.com \\
\hline
\end{tabular}




\begin{tabular}{|c|c|c|}
\hline MCHC (g/dL) & 34.19 & 34.05 \\
\hline Leukocytes (/uL) & 8,100 & 12,600 \\
\hline Basophils (/uL) & 0 & 0 \\
\hline Eosinophils (/uL) & 243 & 378 \\
\hline Band neutrophils (/uL) & 0 & 0 \\
\hline Segmented neutrophils (/uL) & 5,913 & 10,332 \\
\hline Lymphocytes (/uL) & 1,782 & 882 \\
\hline Monocytes (/uL) & 162 & 1008 \\
\hline Platelets & 228,000 & 439,000 \\
\hline Total Plasma Protein (g/dL) & 8.0 & 7.0 \\
\hline Albumin (g/dL) & 2.68 & 2.18 \\
\hline ALT (U/L) & 26 & 23 \\
\hline AST (U/L) & 34 & 72 \\
\hline Creatinine (mg/dL) & 1.21 & 0.86 \\
\hline Alkaline Phosphatase (U/L) & 86 & 106 \\
\hline TPP (g/dL) & 8.93 & 7.18 \\
\hline Urea (g/dL) & 18 & 25 \\
\hline
\end{tabular}

\section{LIST OF FIGURE TITLES}

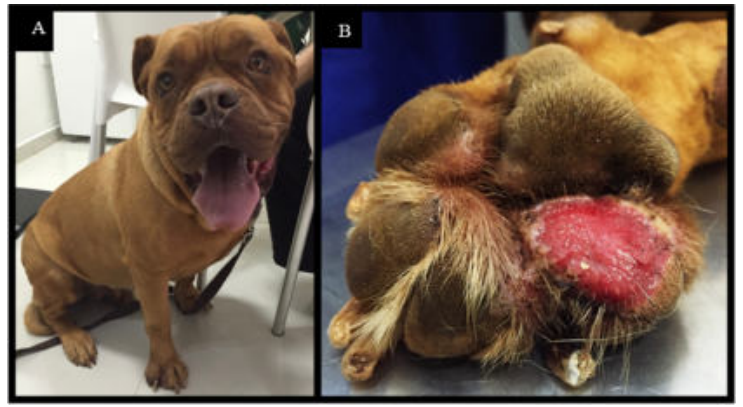

Fig. 1. (A) Female patient, Dogue de Bordeaux. (B) Ulcerated lesion in the fourth digit in the right anterior leg.

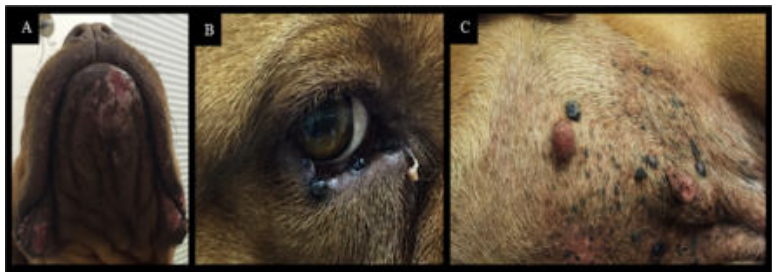

Fig. 2. (A) Ulcerated lesions in the bilateral labial commissure and cranial-mandibular portion. (B) Nodular lesion with a melanocytic pattern in the lower right eyelid. (C) Nodular lesions with a melanocytic pattern disseminated through the abdomen ventral region.

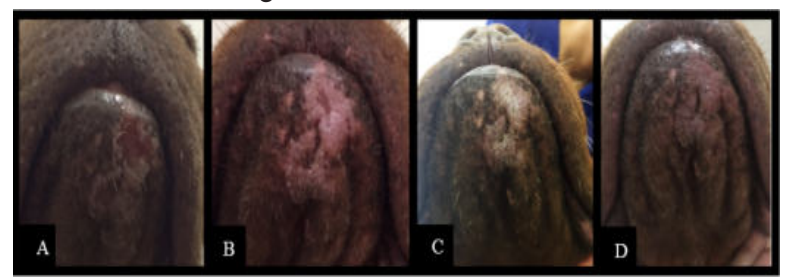

Fig. 3. Healing process of ulcerated lesions in the labial commissures and mandibular region during the Viscum album therapy. (A) Lesion at the initial stage. (B) Lesion stage after one week of treatment.(C) Lesion stage after two weeks of treatment. (D) Lesion stage after four weeks of treatment.

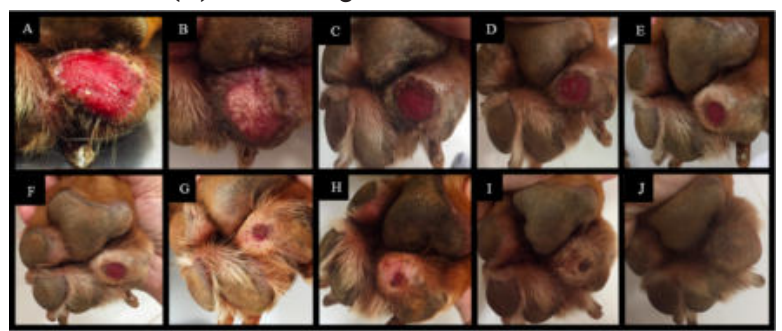

Fig. 4. Healing process of an ulcerated lesion in the fourth digit in the right anterior leg during the Viscum album therapy. (A) Treatment initiation, ulcerated lesion. (B) $1^{\text {st }}$ week after the treatment initiation. (C) $2^{\text {nd }}$ week after the treatment initiation. (D) $3^{\text {rd }}$ week after the treatment initiation. $(E) 4^{\text {th }}$ week after the treatment initiation. (F) $5^{\text {th }}$ week after the treatment initiation. (G) $6^{\text {th }}$ week after the treatment initiation. (H) $7^{\text {th }}$ week after the treatment initiation. (I) $8^{\text {th }}$ week after the treatment initiation. (J) $9^{\text {th }}$ week after the treatment initiation. (K) $10^{\text {th }}$ week after the treatment initiation.

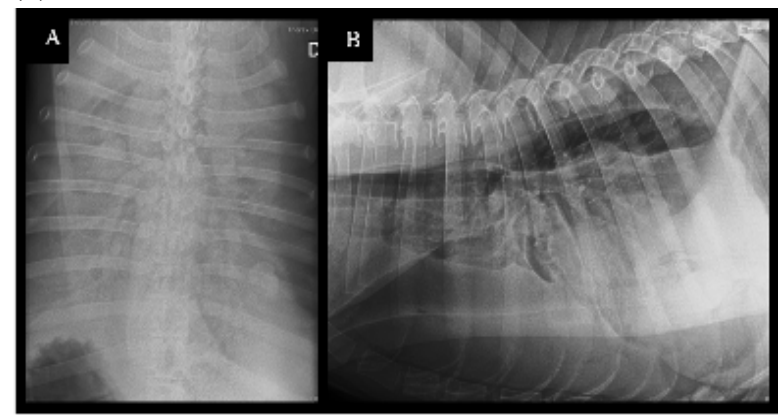

Fig. 5. (A) Dorso-ventral chest $x$-ray, showing pleural effusion. (B) Left lateral chest $x$-ray, evidencing pleural effusion.

\section{REFERENCES}

. Bolon, B., Mays, M.B.C. and Hall, B.J. 1990. Characteristics of Canine Melanomas and Comparison of Histology and DNA Ploidy to Their Biologic Behavior.Vet Pathol.27,96-102.

2. Boston, S.E., Lu, X., Culp, W.T.N., Montinaro, V., Romanelli, G., Dudley, R.M. Liptak,J.M., Mestrinho, L.A. and Buracco, P.2014.Efficacy of systemic adjuvant therapies administered to dogs after excision of oral malignant melanomas: 151 cases (2001-2012). J Am Vet Med Ass. 245(4), 401-407.

3. Ettinger, S. and Feldman, E. 2004. Textbook of veterinary internal medicine: diseases of the dog and the cat. Guanabara Koogan, Rio de Janeiro.

4. Gillard, M., Cadieu, E., Brito, C., Abadie, J., Vergier, B., Devauchelle, P. Degorce, F., Dréano, S., Primot, A., Dorso, L., Lagadic, M., Galibert, F., Hédan, B., Galibert, M.D. and André, C. 2013. Naturally occurring melanomas in dogs as models for non-UV pathways of human melanomas. Pigment Cell Melanoma Res.27(1), 90-102.

5. Grandi, F. and Rondelli, M.C.H. 2016. Cutaneous neoplasms. In Oncology in dogs and cats, Eds., Daleck, C.R. and Nardi, A.B.:Roca, 339-336.

6. Inoue, K., Ohashi, E., Kadosawa, T., Hong, S.H., Matsunaga, S., Mochizuki, M., Nishimura, R. and Sasaki, N. 2004. Establishment and Characterization of Four Canine Melanoma Cell Lines.JVet Med Sci.66(11), 1437-1440.

7. Kaestner, J., Schlodder, D., Preussler, C. and Gruhn, B. 2019. Supportive mistletoe therapy in a patient with metastasised neuroblastoma. BMJ Case Reports. 12(3), 1-5.

8. Kienle, G.S and Kiene, H. 2010. Review article: Influence of Viscum album I (European mistletoe) extracts on quality of life in cancer patients: a systematic review of controlled clinical studies. Integr Cancer Ther . 9, 142157.

9. Lima, S.R., Stocco, M.B., Rondelli, L.A.S., Silva, G.S., Lopes, R.S., Furlan, F.H., Colodel, E.M. and Pescador, C.A. 2018. Cutaneous neoplasms in dogs: 656 cases (2007-2014) from Cuiabá, MT. Pesqui Vet Bras.38(7), 1405-1411.

10. Manley, C.A., Leibman, N.F., Wolchok, J.D., Riviere, I.C., Bartido, S., Craft, D.M. and Bergman, P.J. 2011. Xenogeneic Murine Tyrosinase DNA Vaccine for Malignant Melanoma of the Digit of Dogs.JVet Intern Med. 25, 94-99.

11. Matthes, H., Thronicke, A., Hofheinz, R.D., Baars, E., Martin, D. and Huber, R 2020. Statement to an Insufficient Systematic Review on Viscum album L Therapy. Evid Based Complement Alternat Med. 2020, 1-9.

12. Melo, M.N.O., Oliveira, A.P., Wiecikowski, A.F., Carvalho, R.S., Castro, J.L., Oliveira, F.A.G., Pereira, H.M.G., Veiga, V.F., Capella, M.M.A., Rocha, L. and Holandino, C. 2018. Phenolic compounds from Viscum album tinctures enhanced antitumor activity in melanoma murine cancer cells. Saudi Pharm J. 26(3),311-322.

13. Menke, K., Schwermer, M., Eisenbraun, J., Schramm, A. and Zuzak, T.J. 2021. Anticancer Effects of Viscum album Fraxini Extract on Medulloblastoma Cells in vitro. S. Karger AG. Complement Med Res. 28, 15-22.

14. Menke, K., Schwermer, M., Schramm A. and Zuzak T.J. 2019. Preclinical Evaluation of Antitumoral and Cytotoxic Properties of Viscum album Fraxini Extract on Pediatric Tumor Cells. Planta Med.85(14),1150-1159.

15. Oei, S.L., Thronicke, A., Kröz, M., von Trott, P., Schad, F. and Matthes, H. 2020. Impact of Oncological Therapy and Viscum album $L$ Treatment on CancerRelated Fatigue and Internal Coherence in Nonmetastasized Breast Cancer Patients. Integr Cancer Ther.https://doi.org/10.1177/1534735420917211

16. Rodrigues, A.C., Castro, M.R.,Viscone, E.A., Oliveira, L.A. and Medeiros, A.A. Melanoma with multiple metastasis in dog - case report. 2017. Enciclopédia Biosfera. 14(25), 904-910.

17. Rostock, M. 2020. Die Misteltherapie in der Behandlung von Patienten mit einer Krebserkrankung. Bundesgesundheitsblatt Gesundheitsforschung Gesundheitsschutz. 63,535-540.

18. Siegel, R.L., Miller, K.D. and Jemal, A. 2016. Cancer statistics. CA Cancer J Clin. 66,7-30.

19. Steele, M.L., Axtner, J., Happe, A., Kröz, M., Matthes, H. and Schad, F. 2015. Use and safety of intratumoral application of European mistletoe (Viscum album L) preparations in Oncology. Integr Cancer Ther. 14, 140-148.

20. Strüh, C.M., Jäger, S., Schempp, C.M., Scheffler, A. and Martin, S.F. 2012. A novel triterpene extract from mistletoe induces rapid apoptosis in murine 
B16.F10 melanoma cells. Phytother Res. 10,1507-1512.

21. Sulaimon, S.S., Kitchell, B.E. and Ehrhart, E.J. 2002. Immunohistochemical Detection of Melanoma-specific Antigens in Spontaneous Canine Melanoma. J Comp Pathol. 127, 162-168.

22. Tuohy,J.L., Selmic,L.E.,Worley, D.R., Ehrhart, N.P.,Withrow, S.J.2014. Outcome following curative- intent surgery for oral melanoma in dogs: 70 cases (19982011).J Am Vet Med Assoc 245, 1266-1273.

23. Valle, A.C.V., Andrade, R.V., Sibata, M.N. and Carvalho, A.C. 2020. Ultradiluted Viscum album in the Treatment of Melanoma in a Dog (Canis familiaris) - Case Report. Homeopatia. 109(1), 1-28.

24. Wobeser, B.K., Kidney, B.A., Powers, B.E.,Withrow, S.J., Mayer, M.N., Spinato, M.T. and Allen, A.L. Diagnoses and Clinical Outcomes Associated with Surgically Amputated Canine Digits Submitted to Multiple Veterinary Diagnostic Laboratories. Vet Pathol.44,355-361.

25. Worthmann, P.G., Hintze, A. and Kienle, G.S. 2017. Complete remission and long-term survival of a patient with melanoma metastases treated with highdose fever-inducing Viscum album extract. Medicine.96(46), 1-6. 